\title{
Analysis of Interaction of Surfactant Molecules with $\mathrm{Si}(\boldsymbol{h} \boldsymbol{k l})$ Planes on the Basis of Anisotropic Etching in Alkaline Solutions
}

\author{
I. ZuBEL AND M. KRAMKOWSKA \\ Faculty of Microsystems Electronics and Photonics, Wrocław University of Technology \\ Janiszewskiego 11/17, 50-372 Wrocław, Poland
}

\begin{abstract}
In the paper, measurements of surface tension of solutions used for silicon etching and results of etching in the solutions are presented. Based on the obtained results, the analysis of interactions of surfactants with differently oriented silicon planes has been carried out.
\end{abstract}

PACS numbers: $61.30 . \mathrm{Hn}, 61.72 . \mathrm{uj}, 64.70 . \mathrm{kg}$

\section{Introduction}

In silicon anisotropic etching, tensioactive compounds are often employed as additives to etching solutions in order to improve the morphology of etched surfaces or reduce etching rates of some crystallographic planes. The reduction is advantageous in case of deep etching of complex micro electromechanical systems (MEMS) and micro-opto-electromechanical systems (MOEMS) structures since it enables minimizing of convex corners undercut. The tensioactive compounds like alcohols or typical surfactants reduce etching rates of the planes like (110), (331), (221), causing simultaneously significant improvement of their roughness [1]. A direct assessment of interaction of such compounds with the etched surface is extremely complex as it requires in situ study of etching process in concentrated alkaline solutions. Regular employment of surfactants as the additives, modifying the etching process points out that surface tension of etching solutions may be an important factor characterizing the etching process. The latest studies show that the reduction of surface tension of etching solutions connected with gathering of surfactant molecules at the solution-air interface is directly associated with reduction of wetting angle of the solid by the solution and that the phenomena at the solid-liquid interface are similar to the ones occurring in the solution surface [2]. Studying of surface tension of etching solutions may be an excellent tool for the analysis of the processes occurring on the surface of etched Si.

\section{Experiments}

The results published in the literature concern mainly Si substrates with (100) crystallographic orientation, less frequently with (111) and (110) ones. The surfaces

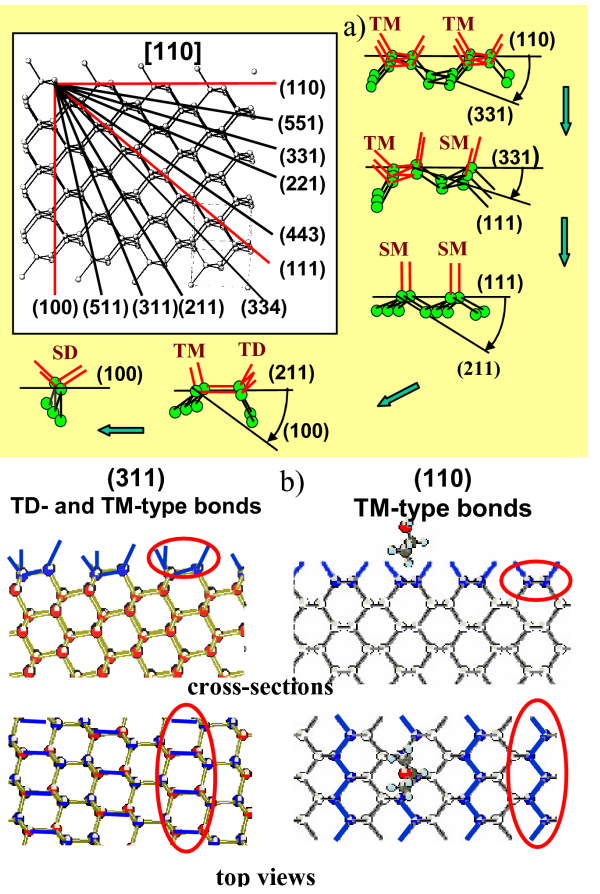

Fig. 1. The bonds on the planes arranged in [110] crystallographic direction: (a) cross-section of Si lattice observed in [110] direction, (b) cross-sections and top views of (311) and (110) planes.

are characterized with extremely different surface bonds which makes the etching results (etching rates and surface roughness) to be completely different. To carry out a comprehensive analysis of etching behaviour, especially the etching behaviour in surfactants containing solutions, the considerations should be extended to vicinal crystallographic orientations with surface bonds being a superposition of the three basic bond types. The bonds on the 
planes from [110] crystallographic zone together with the cross-section of silicon lattice in [110] direction are shown in Fig. 1a and b.

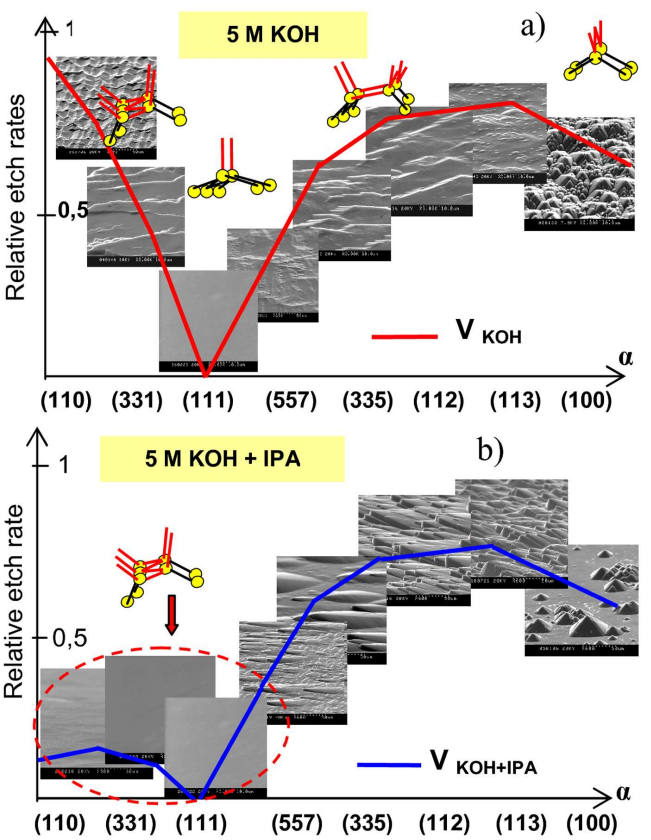

Fig. 2. Comparison of etching rates and roughness of $\mathrm{Si}(h k l)$ surfaces etched in $\mathrm{KOH}$ and $\mathrm{KOH}$ saturated with isopropyl alcohol: (a) $5 \mathrm{M} \mathrm{KOH}$, (b) $5 \mathrm{M}$ $\mathrm{KOH}+$ IPA.

Our earlier studies concerning the etching of Si substrates in $\mathrm{KOH}$ and $\mathrm{KOH}$ saturated with isopropyl alcohol (IPA) revealed that smooth surfaces of (100) and vicinal planes (like for example (311)) can be obtained in etching solutions with the concentrations above $10 \mathrm{M}$ (Fig. 2a). Saturation of the solution with isopropanol results in significant improvement in the roughness of the planes with the bonds classified in the literature as step monohydride (SM) and terrace monohydride (TM), with significant reduction of their etch rates. The planes with the bonds classified as step dihydride (SD) and terrace dihydride (TD) tend to cover with numerous pyramidal structures whereas the alcohol effect on their etching rates is negligible (Fig. 2b).

Systematic studies on IPA and other surfactants (alcohols and diols) effect on the run of etching process were preceded by tensiometric measurements of surface tension of $\mathrm{KOH}$ solutions with surfactant additives (Fig. 3a). The etching processes in the solutions with constant surface tensions $(\delta=50 \mathrm{mN} / \mathrm{m} \mathrm{i} \delta=30 \mathrm{mN} / \mathrm{m})$ and with constant concentrations of surfactant were carried out. The results of etching were assessed based on etching rates and surface roughness. The etching rates of Si planes with different crystallographic orientations in the solutions with selected surfactants are shown in Fig. 3b. The roughness of the planes was evaluated based on scanning electron microscopy (SEM) examinations.
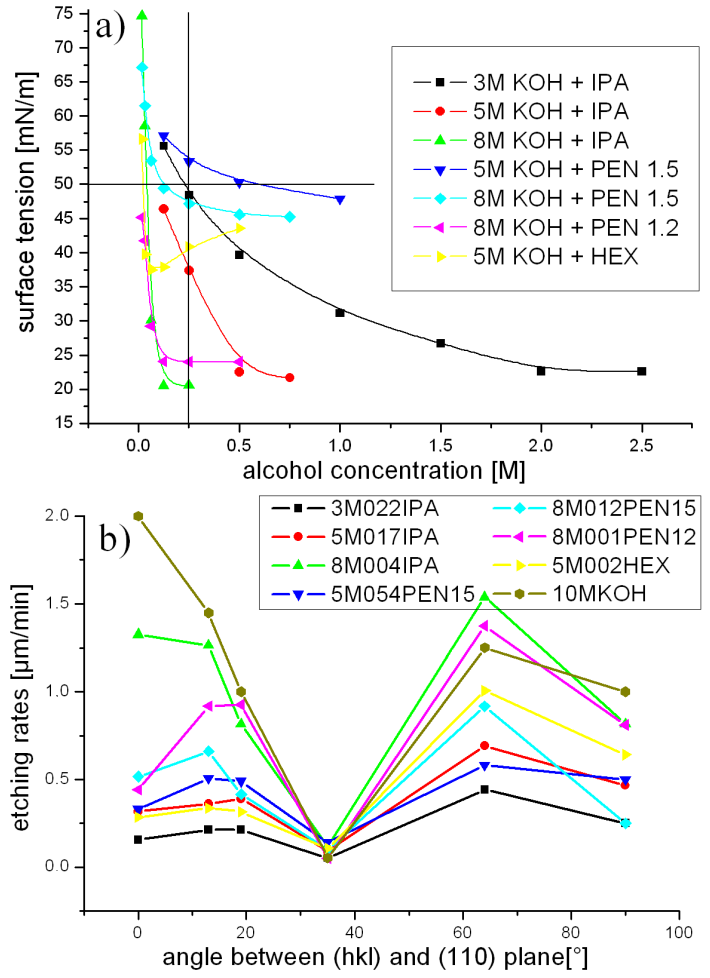

Fig. 3. (a) Surface tensions of etching solutions versus surfactant concentration, (b) etching rates of $\mathrm{Si}(h k l)$ planes in the solutions with selected surfactants at $\delta=50 \mathrm{mN} / \mathrm{m}$.

\section{Analysis of surfactant interaction with surface bonds of $\mathrm{Si}(h k l)$ planes}

Alcohols and diols are non-ionic tensioactive compounds with a hydrophilic and hydrophobic part of carbon chain. Reduction of surface tension of a solution results from gathering of surfactant molecules at the solution surface (surface adsorption) with their hydrophilic part $(\mathrm{OH})$ directed into the solution. The molecules interact with the surface of a silicon substrate immersed in the solution with their hydrophobic part.

In case of almost all examined surfactants, a significant decrease in etching rates of the planes with SM and TM types of bonds and improvement of their roughness in the solutions not saturated with the surfactant have been observed. It can be inferred that some ordering of surfactant molecules, blocking the access of etching agent to the surface is necessary (Fig. 4a). The smoothest surfaces of (110) and (331) oriented substrates were obtained in not saturated solutions with low concentration of $\mathrm{KOH}$ which was connected with low etching rates of the planes and almost isotropic character of etching process.

Etching rates of the planes with TD and SD bonds tended to decrease up to saturation of etching solution with surfactant, however the saturation of the solution was not always obtained. In the saturated solutions, etching rates, especially etching rate of (100) plane, 


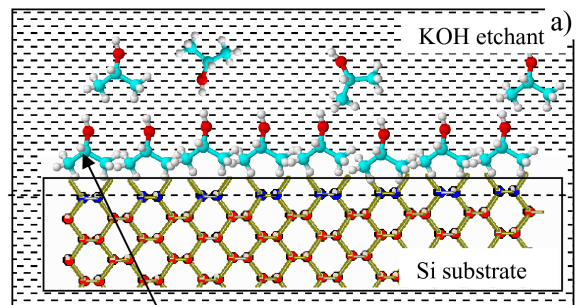

IPA adsorption layer

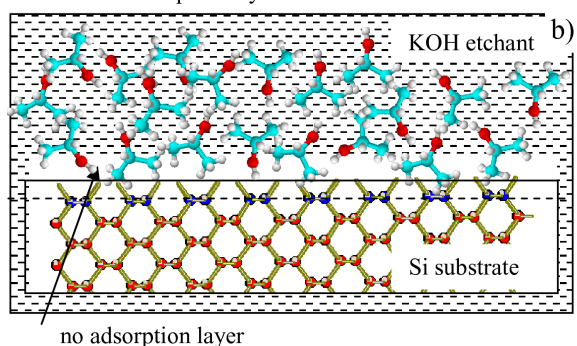

no adsorption layer

Fig. 4. The model of interaction of surfactant molecules with the solution and silicon surface: (a) not saturated solution, (b) saturated solution.

rapidly increased with simultaneous improvement of their morphologies. In the case of these substrates, the lack of an ordered surfactant layer on the etched surface results in higher etching rates and reduction of the number of pyramidal structures (hillocks) on the etched surface (Fig. 4b).

The morphology of etched surfaces (in spite of a constant surface tension) was still dependent on the solution composition. Sole reduction of surface tension to a certain level does not assure appropriate roughness of etched surface. It is necessary to maintain appropriate relation between surface tension and hydroxide concentration, which is connected with the rate of change in surface tension versus surfactant concentration (derivative of $\delta=f(c)$, called surface excess). The phenomenon will be the subject of our further investigations.

\section{Acknowledgments}

This work was supported by the Polish Ministry of Scientific Research and Information Technology grant No. N N515 440533 and Wrocław University of Technology statutory grant.

\section{References}

[1] I. Zubel, M. Kramkowska, Sens. Actuat A 115, 549 (2004).

[2] K. Szymczyk, B. Janczuk, J. Colloid Interface Sci. 303, 319 (2006). 